\title{
The relationship between \\ circulating irisin, retinol binding \\ protein-4, adiponectin and \\ inflammatory mediators in patients \\ with metabolic syndrome
}

Omur Tabak', Gonul Simsek², Fusun Erdenen ${ }^{3}$, Volkan Sozer ${ }^{4}$, Tuna Hasoglu ${ }^{5}$, Remise Gelisgen ${ }^{6}$, Esma Altunoglu ${ }^{4}$, Cuney†

Muderrisoglu ${ }^{4}$, Abdulhalim Senyigit7 , Hafize Uzun ${ }^{6}$

${ }^{1}$ Internal Medicine Clinic,

Istanbul Kanuni Sultan Suleyman

Education and Research

Hospital, Istanbul, Turkey

${ }^{2}$ Department of Physiology,

Istanbul University, Cerrahpasa

Medical Faculty, Istanbul, Turkey

${ }^{3}$ Istanbul Education and

Research Hospital, Internal

Medical Clinic, Istanbul, Turkey

${ }^{4}$ Department of Biochemistry, Yildiz

Technical University, Istanbul, Turkey

${ }^{5}$ Istanbul Medical Faculty, Istanbul

University, Istanbul, Turkey

${ }^{6}$ Department of Biochemistry,

Istanbul University, Cerrahpasa

Medical Faculty, Istanbul, Turkey

${ }^{7}$ Medicine Hospital, Internal

Medical Clinic, Istanbul, Turkey

\section{Correspondence to:}

Hafize Uzun

Department of Medical Biochemistry, Cerrahpasa Faculty of Medicine,

Istanbul University

34303 - Cerrahpasa - Istanbul, Turkey

huzun59@hotmail.com

Received on Dec/20/16

Accepted on May/9/2017

DOI: 10.1590/2359-3997000000289

\section{INTRODUCTION}

$\mathrm{T}$ he metabolic syndrome (MetS), which has an important role in cardiovascular morbidity and mortality, is generally characterized by obesity, insulin resistance (IR), hypertension, cardiovascular disease (CVD), non-alcoholic fatty liver disease and/ or a proinflammatory state due to the accumulation of adipose tissue. Visceral obesity, which is central to MetS, leads to altered adipokines, IR, endothelial dysfunction and atherogenesis. When adipose tissue inflammation and dysfunction are established, adipokine secretion is significantly skewed toward a diabetogenic, proinflammatory and atherogenic pattern $(1,2)$.
Irisin is a novel exercise-mediated myokine that regulates energy metabolism by increasing metabolic rate and mitochondrial content in both myocytes and adipocytes and plays an important role in metabolic diseases. Irisin acts as an exercise-induced insulin sensitizing hormone. The main effects of irisin are the browning of white adipose tissue and increased energy expenditure. Irisin expression and/or circulating levels have been associated with anthropometric and biochemical parameters, other hormones and adipokines, and obesity, IR, type 2 diabetes and MetS $(3,4)$.

Vitamin A is an essential nutrient that is mainly stored in the liver. Retinol binding protein (RBP) is a 
plasma transporter that carries retinol from the liver to the periphery. Very little RBP originates from adipose tissue. RBP-4 is a novel adipokine and a negative acute phase reactant (5). The high concentration of RBP-4 plays a role in the progression of IR through immunity and inflammatory mechanisms in adipose and vascular tissues. RBP-4 seems to be a cardiometabolic marker in chronic inflammatory diseases including obesity, type 2 diabetes, MetS and CVD. These effects result from the direct activation of antigen presenting cells by RBP-4 (6).

Cytokines released by adipose tissue are involved in initiating and promoting a proinflammatory status, which contributes to IR. One of these cytokines, adiponectin, is suggested to sensitize the body to insulin and has a cardioprotective effect (2). Adiponectin is produced by adipocytes and has insulin sensitizing, anti-inflammatory, antioxidative and antiapoptopic properties. Adiponectin enhances insulin secretion by stimulating both the expression of the insulin gene and the exocytosis of insulin granules. Adiponectin also acts in the brain to increase energy expenditure and may thereby promote weight loss. Adiponectin is independently and negatively related to MetS, IR, type 2 diabetes, body weight, blood pressure and serum lipids (7-10).

Pentraxins are divided into the short and long pentraxin families. CRP is the prototype of the short pentraxin subfamily and pentraxin-3 (PTX-3) belongs to the long pentraxin family. PTX-3 is a multimeric acute phase inflammatory glycoprotein in the same family as CRP, a well-known cardiovascular biomarker (11). Its secretion is stimulated in endothelial cells, macrophages, myeloid cells, and dendritic cells by cytokines and endotoxins. After release, PTX-3 binds with Clq to initiate complement activation and facilitate pathogen recognition by macrophages. PTX-3 levels are increased in CVD. Although PTX-3 is an acute inflammatory protein in the same family as CRP, its levels may more directly reflect the inflammatory status of the vasculature $(12,13)$.

Interleukin-33 (also known as IL-1) is a newly identified cytokine. Its receptor, soluble ST-2, has been shown to be protective in certain conditions such as obesity and atherosclerosis. IL-33 exerts protective metabolic effects by decreasing IR, leading to the accumulation of protective Th2 cells and cytokines and consequently reducing adipogenesis $(14,15)$.

We aimed to investigate whether there is a relationship between circulating irisin, RBP-4, PTX-3, IL-33 and adiponectin along with the anthropomorphic and biochemical variables implicated in the development of IR in MetS.

\section{SUBJECTS AND METHODS}

\section{Subjects}

A total of 180 patients and control subjects between the ages of 30-65 were included in the study. MetS was diagnosed according to the Adult Treatment Panel III (ATPIII) criteria by the same physician (16). The components of MetS were waist circumference (WC) > $102 \mathrm{~cm}$ for males and $>88 \mathrm{~cm}$ for females, hypertension (HT) (systolic blood pressure $(\mathrm{SBP}>130 \mathrm{mmHg}$, diastolic blood pressure (DBP) $>85 \mathrm{mmHg}$ ), treatment with antihypertensive drugs, high density lipoproteincholesterol (HDL-C) $<40 \mathrm{mg} / \mathrm{dL}$ in males and < $50 \mathrm{mg} / \mathrm{dL}$ in females, hypertriglyceridemia $(\mathrm{TG})>150$ $\mathrm{mg} / \mathrm{dL}$, fasting blood glucose $(\mathrm{FBG})>100 \mathrm{mg} / \mathrm{dL}$ or the presence of type 2 diabetes mellitus (DM). MetS was defined as the presence of at least three components. Pregnant women, subjects with endocrine disorders, chronic cardiovascular, renal, hepatic, rheumatic diseases, smokers and subjects who were taking drugs which could affect our results were excluded. The control group consisted of 50 healthy people who were hospital staff. This group did not have diabetes, dyslipidemia or glucose intolerance as confirmed by an oral glucose tolerance test (OGTT). They neither had hepatic nor renal disease and were not taking drugs to affect carbohydrate metabolism. Pregnant women, patients with acute vascular or infectious illness or malignancy were also excluded. All subjects were of Turkish descent. The weight and height of each person were measured, and body mass index (BMI) was calculated according to the following formula: weight/ height $\left(\mathrm{m}^{2}\right)$. Waist circumference was measured with a flexible tape measurer at the level of the navel.

All participants were informed about the survey and freely signed and dated the consent form. The protocol was approved by the Ethics Committee of Istanbul Education and Research Hospital and was conducted in accordance with the Declaration of Helsinki (No: 2015/628).

\section{Laboratory analysis}

\section{Sample collection and preparation}

Drugs were administered at least $24 \mathrm{~h}$ prior to blood collection. Clinical parameters, including routine biochemical parameters, were measured using standard protocols. Blood samples were collected in EDTA- 
containing tubes and anticoagulant-free tubes after an overnight fast. After immediate centrifugation (3000 g) for $10 \mathrm{~min}$ at $4{ }^{\circ} \mathrm{C}$, plasma and serum were separated in Eppendorf tubes and frozen immediately at $-80{ }^{\circ} \mathrm{C}$ until analysis.

The homeostasis model assessment (HOMA) was used to detect the degree of insulin resistance (IR) by measuring the levels of basal (fasting) glucose and insulin. HOMA-IR was calculated using the following formula: HOMA-IR = (fasting glucose $[\mathrm{mg} / \mathrm{dL}] \mathrm{x}$ fasting insulin $[\mu \mathrm{U} / \mathrm{mL}]) / 405$.

\section{Measurement of plasma pentraxin-3 (PTX3) concentrations}

Plasma PTX-3 levels were measured by a commercially available competitive enzyme-linked immunoassay kit (Hycult Biotech, Netherlands, Catno; HK347). The coefficients of intra- and inter-assay variation were $4.4 \%$ $(\mathrm{n}=15)$ and $5.5 \%(\mathrm{n}=15)$, respectively.

\section{Measurement of serum interleukin-33 (IL-33) concentrations}

Serum Il-33 levels were measured by a commercially available competitive enzyme-linked immunoassay kit (eBiosience, Austria, BMS2048). The coefficients of intra- and inter-assay variation were $5.0 \%(\mathrm{n}=15)$ and $6.5 \%(\mathrm{n}=15)$, respectively.

\section{Measurement of serum irisin concentrations}

Serum irisin levels were measured by a commercially available competitive enzyme-linked immunoassay kit (EASTBIOPHARM, Hangzhou Eastbiopharm Co. Ltd. China). The coefficients of intra- and interassay variation were $6.7 \%(\mathrm{n}=15)$ and $7.5 \%(\mathrm{n}=15)$, respectively.

\section{Measurement of serum retinol binding protein-4 (RBP-4)}

Serum RBP-4 levels were measured by a commercially available competitive enzyme-linked immunoassay kit (EASTBIOPHARM, Hangzhou Eastbiopharm Co. Ltd. China). The coefficients of intra- and interassay variation were $6.4 \%(\mathrm{n}=15)$ and $7.2 \%(\mathrm{n}=15)$, respectively.

\section{Measurement of serum adiponectin concentrations}

Serumadiponectin levelswere measured by a commercially available sandwich enzyme-linked immunoassay kit
(DRG International, Inc., Marburg, Germany). The coefficients of intra- and inter-assay variation were $6.3 \%$ $(\mathrm{n}=15)$ and $7.3 \%(\mathrm{n}=15)$, respectively.

Glucose, TC, TG, HDL-C and LDL-C concentrations were determined by enzymatic methods (Abbott Diagnostics, Abbott Park, IL, USA). The intra-assay and inter-assay coefficients of variation were $2.6 \%$ and $3.0 \%$ for the assayed glucose, respectively, $2.6 \%$ and $3.0 \%$ for the total cholesterol assayed, respectively, $2.7 \%$ and $3.5 \%$ for the assayed TG, respectively, $3.5 \%$ and $4.1 \%$ for the assayed HDL-C, respectively, and $3.9 \%$ and $4.2 \%$ for the assayed LDL-C, respectively. Insulin concentrations were measured by an electrochemiluminescence immunoassay (ECLIA) method on a Roche-Hitachi E170. IR was calculated using the homeostasis model assessment formula (HOMA-IR, fasting insulin $(\mathrm{mU} / \mathrm{L}) *$ glucose $(\mathrm{mmol} / \mathrm{dL} / 22.5)$. Analysis of CRP was performed by nephelometric means (IMAG-Bechman Coulter, Krefeld, Germany).

\section{Statistical analysis}

Statistical analyses were performed using SPSS 20.0 for Windows (SPSS, Inc., Chicago, Illinois). The results are expressed as the mean \pm standard deviation. An independent samples t-test was used to compare the mean values between the groups. Spearman's rho test was used to determine the correlations with MetS risk factors. Pearson's correlation was used for numerical data. In addition, a linear regression analysis was conducted to determine the parameters that significantly affect the MetS. Chi square test was performed to observe the quantitative similarity between genders. To assess the diagnostic accuracy, we performed receiver operating characteristic (ROC) curve analysis. ROC analysis was performed using MedCalc Statistical Software version 14.8.1 ((MedCalcSoftware bvba, Ostend, Belgium). The area under the ROC curve (AUC) was then estimated. $p<$ 0.05 values were considered to be statistically significant.

\section{RESULTS}

The demographic and biochemical values of MetS patients and controls are shown in Table l. Age and sex matched groups revealed significant differences with regard to their demographic and biochemical values, as expected (Table 1). The comparison of irisin, retinol binding protein-4, adiponectin and inflammatory 
mediators of the groups according to $\mathrm{HbAlc}$ levels are shown in Table 2. Circulating irisin, RBP-4, adiponectin and inflammatory mediators of are shown in Table 3.
Correlation analysis between biochemical variables of the whole group and the MetS group are shown in the Tables 4 and 5 .

Table 1. Demographic and biochemical values of metabolic syndrome patients and controls

\begin{tabular}{|c|c|c|c|}
\hline & Control group $(n=50)$ & Metabolic syndrome $(n=130)$ & $P$ \\
\hline Age & $48.72 \pm 5.98$ & $50.29 \pm 5.67$ & NS \\
\hline $\operatorname{Sex}(F / M)$ & $26 / 24$ & $68 / 62$ & NS \\
\hline Waist circumference (cm) & $82.62 \pm 9.13$ & $102.87 \pm 8.21$ & $<0.001$ \\
\hline Hip circumference (cm) & $96.82 \pm 8.76$ & $113.60 \pm 9.19$ & $<0.001$ \\
\hline BMl $\left(\mathrm{kg} / \mathrm{m}^{2}\right)$ & $23.86 \pm 2.40$ & $33.54 \pm 4.35$ & $<0.001$ \\
\hline Systolic Blood Pressure (mmHg) & $114.80 \pm 8.06$ & $137.08 \pm 11.71$ & $<0.001$ \\
\hline Diastolic Blood Pressure (mmHg) & $78.40 \pm 8.57$ & $85.23 \pm 8.06$ & $<0.001$ \\
\hline Total protein (g/dL) & $7.32 \pm 0.40$ & $7.46 \pm 0.36$ & NS \\
\hline Albumin (g/dL) & $4.54 \pm 0.29$ & $4.42 \pm 0.30$ & NS \\
\hline Uric acid (mg/dL) & $3.94 \pm 1.00$ & $5.35 \pm 1.57$ & $<0.001$ \\
\hline Total cholesterol (mg/dL) & $162.50 \pm 24.88$ & $201.86 \pm 46.22$ & $<0.001$ \\
\hline HDL-C (mg/dL) & $49.68 \pm 9.52$ & $46.18 \pm 11$ & $<0.05$ \\
\hline LDL-C (mg/dL) & $97.84 \pm 24.35$ & $110.98 \pm 37.49$ & $<0.01$ \\
\hline Triglyceride (mg/dL) & $94.00 \pm 30.33$ & $210.82 \pm 112.08$ & $<0.001$ \\
\hline Fibrinogen (mg/dL) & $260.54 \pm 54.07$ & $330.68 \pm 69.26$ & $<0.001$ \\
\hline Glucose (mg/dL) & $91.38 \pm 9.29$ & $177.15 \pm 67.89$ & $<0.001$ \\
\hline C-peptide (ng/mL) & $1.73 \pm 0.61$ & $2.22 \pm 1.14$ & $<0.01$ \\
\hline $\mathrm{HbA1}_{\mathrm{C}}(\%)$ & $5.51 \pm 0.44$ & $8.10 \pm 1.73$ & $<0.001$ \\
\hline Insulin ( $\mu \mathrm{U} / \mathrm{mL})$ & $9.51 \pm 5.03$ & $27.45 \pm 30.79$ & $<0.001$ \\
\hline HOMA-IR & $2.16 \pm 1.20$ & $12.32 \pm 16.82$ & $<0.001$ \\
\hline
\end{tabular}

BMI: body mass index; LDL: low density lipoprotein; HDL: high density lipoprotein; NS: non-significant.

Table 2. Comparison of irisin, retinol binding protein-4, adiponectin and inflammatory mediators of the groups according to HbA1c levels

\begin{tabular}{lccccc} 
& $\begin{array}{c}\text { Group A } \\
\text { (HbA1c }<\mathbf{6 . 5 \% )} \\
\mathbf{n}=\mathbf{6 8}\end{array}$ & $\begin{array}{c}\text { Group B } \\
\text { (HbA1c: } \mathbf{6 . 5 - 8 \% )} \\
\mathbf{n}=\mathbf{5 6}\end{array}$ & $\begin{array}{c}\text { Group C } \\
\text { (HbA1c > 8\%) } \\
\mathbf{n}=\mathbf{5 6}\end{array}$ & P (A-B) & P (A-C) \\
\hline hsCRP $(\mathrm{mg} / \mathrm{dL})$ & $0.97 \pm 1.37$ & $2.97 \pm 1.63$ & $3.19 \pm 1.72$ & $\mathbf{0 . 0 0 0}$ & $\mathbf{0 . 0 0 0}$ \\
PTX-3 $(\mathrm{pg} / \mathrm{mL})$ & $256.89 \pm 120.27$ & $326.53 \pm 107.10$ & $317.83 \pm 112.30$ & $\mathbf{0 . 0 0 1}$ & $\mathbf{0 . 0 0 5}$ \\
IL-33 $(\mathrm{pg} / \mathrm{mL})$ & $5.18 \pm 1.14$ & $5.23 \pm 0.82$ & $5.37 \pm 0.86$ & 0.793 & 0.325 \\
Irisin $(\mathrm{ng} / \mathrm{mL})$ & $47.95 \pm 19.05$ & $62.61 \pm 18.07$ & $62.56 \pm 17.23$ & $\mathbf{0 . 0 0 0}$ & $\mathbf{0 . 0 0 0}$ \\
RBP- $4(\mathrm{mg} / \mathrm{mL})$ & $40.35 \pm 12.28$ & $54.49 \pm 10.71$ & $57.64 \pm 15.51$ & $\mathbf{0 . 0 0 0}$ & $\mathbf{0 . 0 0 0}$ \\
Adiponectin $(\boldsymbol{\mu g} / \mathrm{mL})$ & $6.13 \pm 2.14$ & $3.61 \pm 1.27$ & $3.46 \pm 1.30$ & $\mathbf{0 . 0 0 0}$ & $\mathbf{0 . 0 0 0}$ \\
\hline
\end{tabular}

hsCRP: high sensitivity C-reactive protein; PTX-3: pentraxin-3; IL-33: interleukin-33; RBP- 4: retinol binding protein - 4 .

There was no difference between Group B and $\mathrm{C}$ with regard to these biomarkers.

Table 3. Circulating irisin, retinol binding protein-4, adiponectin and inflammatory mediators of MetS and controls

\begin{tabular}{lcc}
\hline & Control group & Metabolic syndrome \\
$\mathbf{n = 5 0}$ & $\mathbf{n}=\mathbf{1 3 0}$ & $3.04 \pm 1.67$ \\
\hline $\operatorname{hsCRP}(\mathrm{mg} / \mathrm{dL})$ & $0.35 \pm 0.24$ & $321.46 \pm 110.63$ \\
PTX-3 $(\mathrm{pg} / \mathrm{mL})$ & $235.29 \pm 111.08$ & $5.35 \pm 0.88$ \\
IL-33 $(\mathrm{pg} / \mathrm{mL})$ & $5.02 \pm 1.12$ & $6.35 \pm 1.75$ \\
Irisin $(\mathrm{ng} / \mathrm{mL})$ & $4.03 \pm 1.29$ & $56.13 \pm 12.83$ \\
RBP-4 $(\mathrm{ng} / \mathrm{mL})$ & $34.55 \pm 6.63$ & $<.001$ \\
Adiponectin $(\mu \mathrm{g} / \mathrm{mL})$ & $7.03 \pm 1.71$ & $3.56 \pm 1.23$ \\
\hline
\end{tabular}

hsCRP: high sensitivity C-reactive protein; PTX-3: pentraxin-3; IL-33: interleukin-33; RBP- 4: retinol binding protein - 4. 
Table 4. Correlation analysis of groups

\begin{tabular}{|c|c|c|c|c|c|c|c|c|}
\hline & \multicolumn{2}{|c|}{ Irisin } & \multicolumn{2}{|c|}{ Adiponectin } & \multicolumn{2}{|c|}{ RBP-4 } & \multicolumn{2}{|c|}{ PTX-3 } \\
\hline & & & $r$ & $p$ & $r$ & $p$ & $r$ & $p$ \\
\hline SBP & & & -0.55 & $<0.001$ & 0.467 & 0.0001 & & \\
\hline \multicolumn{9}{|l|}{ DBP } \\
\hline BMl & 0.36 & $<0.001$ & 0.34 & $<0.001$ & 0.479 & 0.0001 & 0.229 & $<0.002$ \\
\hline WC & 0.49 & $<0.001$ & 0.54 & $<0.001$ & 0.474 & $<0.0001$ & 0.229 & $<0.002$ \\
\hline WHR & 0.34 & $<0.001$ & 0.23 & 0.02 & & & & \\
\hline hsCRP & 0.30 & $<0.001$ & & & & & & \\
\hline $\mathrm{HbA1C}$ & 0.28 & $<0.001$ & 0.49 & $<0.001$ & 0.487 & $<0.0001$ & 0.221 & 0.003 \\
\hline Glucose & & & 0.47 & $<0.001$ & 0.51 & $<0.0001$ & & \\
\hline Insulin & & & 0.24 & $<0.001$ & & & & \\
\hline HOMA-IR & & & 0.26 & $<0.001$ & 0.258 & $<0.0001$ & & \\
\hline Cholesterol & & & 0.40 & $<0.001$ & 0.656 & $<0.0001$ & 0.508 & $<0.0001$ \\
\hline LDL-C & 0.01 & 0.88 & 0.29 & $<0.001$ & 0.596 & $<0.0001$ & 0.555 & $<0.0001$ \\
\hline Uric acid & 0.19 & 0.01 & & & 0.559 & $<0.0001$ & 0.416 & $<0.0001$ \\
\hline Fibrinogen & & & 0.36 & $<0.001$ & 0.349 & $<0.0001$ & & \\
\hline PTX-3 & 0.16 & 0.16 & 0.34 & $<0.001$ & 0.512 & $<0.0001$ & & \\
\hline RBP-4 & 0.25 & 0.001 & & & & & & \\
\hline Irisin & & & 0.36 & $<0.001$ & 0.253 & $<0.001$ & & \\
\hline
\end{tabular}

Table 5. Correlation analysis in MetS patients

\begin{tabular}{lcc}
\hline & $\mathbf{r}$ & $\boldsymbol{p}$ \\
\hline Uric acid-Cholesterol & 0.541 & 0.0001 \\
Uric acid-LDL-C & 0.606 & 0.0001 \\
Uric acid-hSCRP & 0.454 & 0.0001 \\
Uric acid-PTX-3 & 0.414 & 0.0001 \\
Uric acid-PBB-4 & 0.485 & 0.0001 \\
Uric acid-Adiponectin & -0.246 & 0.0001 \\
LDL-hsCRP & 0.652 & 0.0001 \\
LDL-PTX-3 & 0.638 & 0.0001 \\
LDL-RBP & 0.694 & 0.0001 \\
LDL-Adiponectin & -0.323 & 0.0001 \\
hsCRP-PTX-3 & 0.472 & 0.0001 \\
hsCRP-RBP-4 & 0.521 & 0.0001 \\
hsCRP-Adiponectin & -0.219 & 0.012 \\
PTX-3-RBP4 & 0.487 & 0.0001 \\
PTX-3-Adiponectin & -0.373 & 0.0001 \\
RBP-4-Adiponectin & -0.215 & 0.014 \\
Irisin-WC & 0.246 & 0.005 \\
Irisin-WHR & 0.468 & 0.0001 \\
Adiponectin-SBP & -0.208 & 0.017 \\
\hline & &
\end{tabular}

Regression analysis for irisin revealed that only WC had a significant effect on MetS $(\mathrm{p}=0.02)$. WHR, BMI, HOMA-IR, uric acid, LDL-C, hsCRP, PTX-3, IL-33, RBP-4, and adiponectin levels did not correlate with irisin. Adiponectin levels did not correlate with these parameters in MetS. Correlation analyses of the new biomarkers are shown in Table 6 .
A comparison of the ROC curves with sensitivity, specificity, AUC, cut-off and asymptotic significance of systolic BP, DBP, WC, glucose, TG, HDL-C, hsCRP, irisin, RBP-4, PTX-3, adiponectin and IL-33 levels in the whole group are shown in Table 7 and in ROC curves in Figures 1 and 2.

\section{DISCUSSION}

Adipose tissue is an important source of adipokines that have proinflammatory effects and may be the link between obesity, CVD, diabetes and $\operatorname{MetS}(1,2)$. In our study, there was a statistically significant difference between the MetS group and the control group with respect to WC, WHR, BMI, blood pressure, lipid parameters, uric acid, fibrinogen, glucose, insulin, $\mathrm{C}$-peptide and HbAlc values. Some of these variables are components of MetS, so this is not surprising. More importantly, there were significant differences with regard to irisin, RBP-4, IL-33, PTX-3 and hsCRP levels in favor of the MetS group; the opposite finding was observed for adiponectin.

We found that circulating irisin levels were positively associated with MetS components including BMI, WC and WHR, while in some studies, there were conflicting results regarding the relation between irisin levels with metabolic parameters. We also observed that women had lower levels of plasma irisin levels compared to men. In the control group, although women had 
Table 6. Correlation analysis of the new parameters with each other

\begin{tabular}{|c|c|c|c|c|c|c|c|}
\hline & & & Irisin & PTX-3 & RBP-4 & Adiponectin & hsCRP \\
\hline \multirow[t]{6}{*}{ MetS } & RBP-4 & $r$ & & 0.487 & & -0.215 & 0.521 \\
\hline & & $p$ & & 0.0001 & & 0.014 & $<0.0001$ \\
\hline & PTX-3 & $r$ & & & & -0.373 & 0.472 \\
\hline & & $p$ & & & & $<0.0001$ & $<0.0001$ \\
\hline & Adiponectin & $r$ & & & & & -0.219 \\
\hline & & $p$ & & & & & 0.012 \\
\hline \multirow[t]{10}{*}{ Whole } & Irisin & $r$ & & & 0.253 & -0.363 & 0.299 \\
\hline & & $p$ & & & 0.001 & 0.0001 & 0.0001 \\
\hline & RBP-4 & $r$ & & 0.512 & & -0.567 & 0.702 \\
\hline & & $p$ & & 0.0001 & & 0.0001 & 0.0001 \\
\hline & PTX-3 & $r$ & 0.157 & & & -0.339 & 0.502 \\
\hline & & $p$ & 0.035 & & & 0.0001 & 0.0001 \\
\hline & Adiponectin & $r$ & -0.363 & -0.339 & & & -0.566 \\
\hline & & $p$ & 0.0001 & 0.0001 & & & 0.0001 \\
\hline & IL-33 & $r$ & & 0.153 & & & \\
\hline & & $p$ & & 0.041 & & & \\
\hline
\end{tabular}

Table 7. Sensitivity, specificity, cut-off, AUC and asymptotic significance of parameters

\begin{tabular}{lccccccc}
\hline & Sensitivity (\%) & Specificity (\%) & Cut-off & AUC & SE & 95\% CI & Asymptotic sig. \\
\hline Systolic BP & 90.00 & 90.00 & $>120$ & 0.938 & 0.016 & $0.892-0.968$ & $<0.0001$ \\
BMI & 96.15 & 94.00 & $>27.18$ & 0.980 & 0.010 & $0.947-0.995$ & $<0.0001$ \\
HbA1C & 87.69 & 98.00 & $>6.2$ & 0.958 & 0.013 & $0.918-0.982$ & $<0.0001$ \\
HOMA-IR & 93.08 & 80.00 & $>2.68$ & 0.927 & 0.019 & $0.878-0.960$ & $<0.0001$ \\
hSCRP & 85.38 & 100.00 & $>0.93$ & 0.975 & 0.009 & $0.940-0.992$ & $<0.0001$ \\
RBP-4 & 81.54 & 98.00 & $>44.56$ & 0.945 & 0.015 & $0.901-0.973$ & $<0.0001$ \\
\hline
\end{tabular}

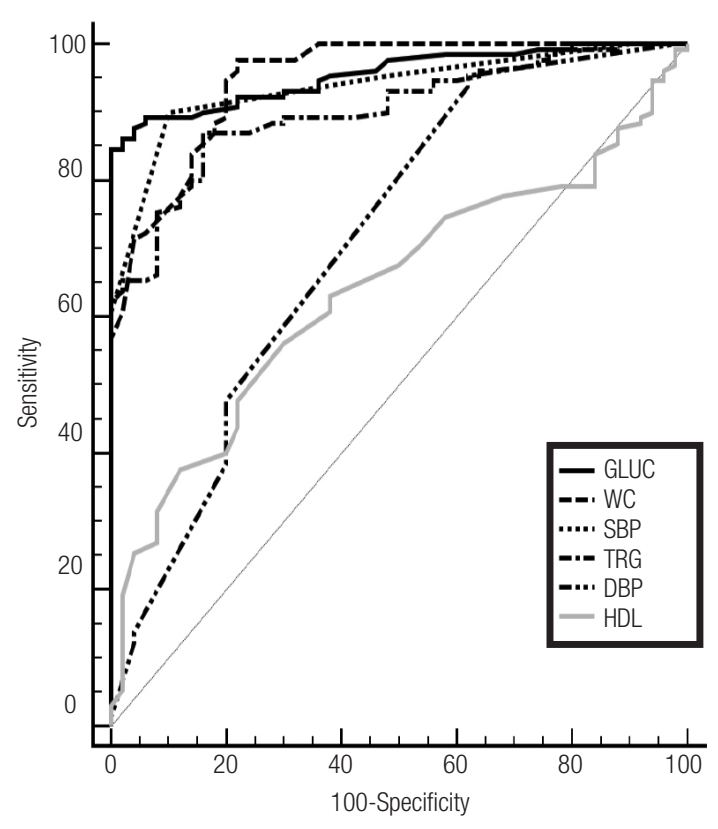

Figure 1. ROC curves of MetS parameters.

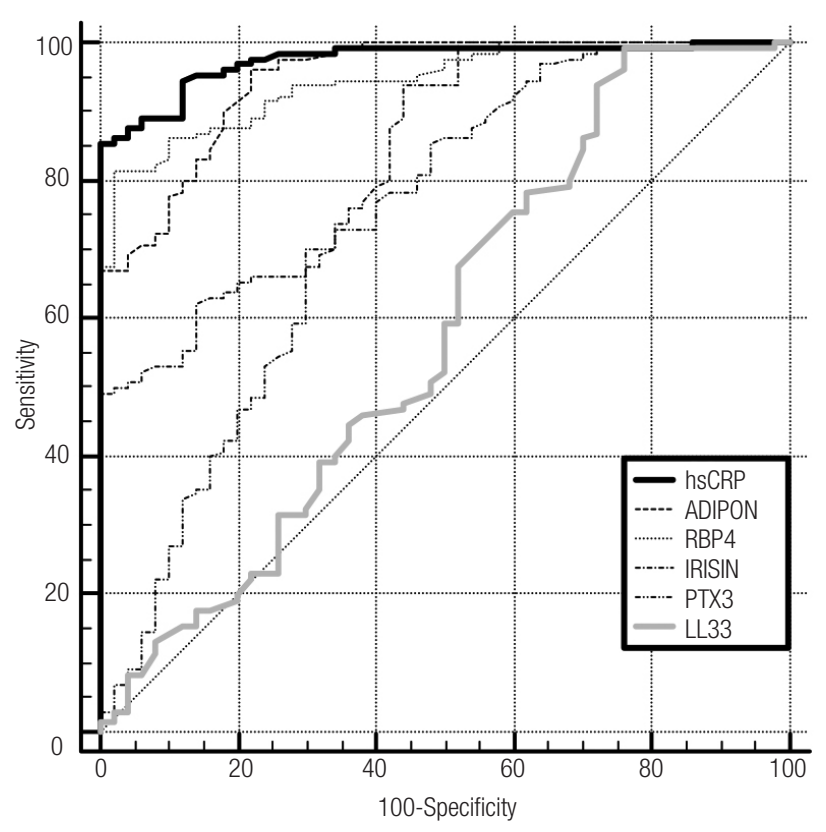

Figure 2. ROC curves of the new biomarkers. 
higher levels, this was not statistically significant. We do not know the mechanism underlying the difference between the two genders. We suggest that this may be due to increased muscle mass, hormonal effects, the duration of obesity or drugs. hsCRP, IL-33, PTX-3, RBP-4 and fibrinogen levels did not change between males and females in the MetS group. Among other variables, only HDL-C levels were different between the two genders, as expected. Rizk and cols. (17) found that irisin levels were increased and positively correlated with BMI, serum triglycerides, HOMA-IR and liver enzymes in patients with MetS. Some authors showed a positive association between irisin levels and plasma glucose and TG levels in type 2 diabetic patients $(18,19)$. Contradictory to these studies, Assyov and cols. (20) found a negative relationship with fasting glucose levels, although he observed a positive association of irisin with BMI. Wang and $\mathrm{Li}(2 \mathrm{l}$ ) reported decreased irisin levels in patients with type 2 DM compared with nondiabetics and suggested that irisin was not associated with beta cell function (21). We did not observe a positive relationship between irisin with plasma glucose, insulin and cholesterol levels. In a cohort of sedentary women, Duran and cols. (22) observed a negative correlation between irisin and BMI, postprandial glucose, LDL-C and TG levels, but a positive relationship with HDL-C. We suggest that irisin might be an early marker of MetS which only affected WC and WHR, but did not influence blood pressure and biochemical parameters.

IL-6 is secreted from adipose tissue in obesity and circulating levels of CRP are increased through adipocyte-derived IL-6 (23). We showed that hsCRP levels were positively correlated with PTX-3 and RBP-4, which are molecules involved in inflammatory conditions; hsCRP was negatively correlated with adiponectin. Inflammation in the vasculature might be an important pathogenic link between CVD and MetS. In our study, we found significantly higher levels of PTX-3 in MetS patients than in control subjects. There was a positive association between PTX-3 and irisin, RBP-4 levels and MetS components; a negative relationship was found between PTX-3 and adiponectin levels. A normal PTX-3 concentration was found to be approximately $2 \mathrm{ng} / \mathrm{mL}$; men had significantly lower values than women $(12,13)$. There was no difference between genders regarding PTX-3 levels in our study. We found an association of PTX-3 with risk factors such as obesity, uric acid, LDL-C, hsCRP, RBP-4 and adiponectin. Inoue and cols. (12) showed that the PTX-3 level was increased in the oldest age group and was also inversely correlated with triglyceride levels and BMI. It has also been found to be independent of other established coronary risk factors (13). Kardas and cols. (24) reported that PTX-3 levels were higher in obese children and adolescents with MetS and CVD, and that these levels were positively correlated with TG and negatively correlated with HDL-C levels $(24,25)$. It was suggested that PTX3 levels may increase in order to confer protection against cardiac tissue damage. PTX3 binds to activated platelets and reduces inflammation in the cardiovascular bed (12), and might be a novel marker for subclinical atherosclerosis (25). These findings suggested that both PTX-3 and RBP-4 may be used to predict inflammatory status in MetS instead of hsCR, which is a well- known acute phase inflammatory marker. In accordance with some studies, we found that RBP-4 levels were higher in obese subjects than controls. We observed that RBP-4 was positively correlated with uric acid, LDL-C, hsCRP, and PTX-3, and negatively related to adiponectin. The concentration of RBP-4 was found to be elevated in obesity and type $2 \mathrm{DM}$, MetS and CVD (5). Many authors found that RBP-4 levels were associated with MetS parameters and HOMA-IR, the duration of diabetes and carotid atherosclerosis as determined by CIMT (26-29). There are conflicting results about the association of RBP-4 and CRP levels, probably due to methodological differences $(30,31)$. Jialal and cols. suggested that serum irisin and RBP-4 levels would be independent predictors of CVD in diabetes (30). We think that RBP-4 is significantly associated with nearly all components of MetS and inflammation. It should be kept in mind that circulating RBP concentrations depend on vitamin A status; therefore, the serum retinol concentration may be a confounder (5). We did not measure vitamin A levels.

As expected, we found lower levels of adiponectin in MetS patients than controls. Adiponectin levels were negatively associated with SBP, WC, WHR, PTX-3, RBP4, hsCRP, uric acid and LDL-C. Bidulescu and cols. (32) observed higher levels of adiponectin in African American women compared to men. Chiara and cols. (33) showed that subjects with low adiponectin levels had a higher prevalence of obesity, MetS, DM and CVD.

In our research, we found IL-33 levels were associated with HbAlc and insulin in the control group, but there no association of this marker with 
other variables. Interestingly, there was no association between IL-33 and adiponectin. IL-33 might be either proinflammatory or anti-inflammatory depending on the disease and the model. However, IL-33 was shown to have various protective effects in CVD, obesity and diabetes. Reduced levels may increase the risk of developing insulin resistance $(14,15)$.

A strength of our study is that we evaluated multiple biomarkers involved in MetS and searched for the relationship between each of them with anthropomorphic and biochemical variables. However, our study has some limitations. First, our sample size is relatively small. Second, dietary habits, physical activity and the exercise level of the subjects were not documented. Third, we did not investigate cardiovascular comorbidities and drugs which could affect our results. Last, we did not measure vitamin A levels which might affect the RBP-4 level. Due to the cross-sectional design of our study, we cannot make any suggestions about the association between the laboratory and clinical parameters of the subjects. Prospective trials are needed to observe this relationship. We think that obesity induced cytokines initiate and promote a proinflammatory status leading to clinical consequences such as IR, DM, HT and atherosclerosis. Discrepancies in our results from previous studies may be due to different study populations or differences in their diet and exercise.

MetS is associated with impaired glucose homeostasis and low-grade chronic inflammation, as well as myokines and adipokines that interact through complex networks. Although the precise mechanisms are still unclear, elevated PTX-3, RBP-4, IL-33, irisin and decreased adiponectin levels increase the risk of obesity-related metabolic disorders. The inflammatory condition associated with overweight plays an important role in the components of the MetS and largely contributes to the related pathological outcomes. Our findings suggested that irisin might be an early marker of MetS that emerges before anthropomorphic, biochemical and clinical parameters. We also suggest that both PTX-3 and RBP-4 may be used to predict the inflammatory status in MetS instead of hsCR, which is a well-known acute phase inflammatory marker. The contradictory results between this study and others may be linked to the different stages of the MetS. Subjects should be evaluated prospectively with anthropomorphic, biochemical and clinical aspects at regular intervals. Future clinical studies are needed to confirm and extend these data.
Disclosure: no potential conflict of interest relevant to this article was reported.

\section{REFERENCES}

1. Ritchie SA, Connell JM. The link between abdominal obesity, metabolic syndrome and cardiovascular disease. Nutr Metab Cardiovasc Dis. 2007;17:319-26.

2. Freitas Lima LC, Braga VA, do Socorro de França Silva M, Cruz JC, Sousa Santos SH, de Oliveira Monteiro MM, Balarini CM. Adipokines, diabetes and atherosclerosis: an inflammatory association. Front Physiol. 2015;6:304.

3. Panagiotou G, Mu L, Na B, Mukamal KJ, Mantzoros CS. Circulating irisin, omentin-1, and lipoprotein subparticles in adults at higher cardiovascular risk. Metabolism. 2014;63:1265-71.

4. Vaughan RA, Gannon NP, Mermier CM, Conn CA. Irisin, a unique non-inflammatory myokine in stimulating skeletal muscle metabolism. J Physiol Biochem. 2015;71:679-89.

5. Zabetian-Targhi F, Mahmoudi MJ, Rezaei N, Mahmoudi M. Retinol Binding Protein 4 in Relation to Diet, Inflammation, Immunity, and Cardiovascular Diseases. Adv Nutr. 2015;6:748-62.

6. Moraes-Vieira PM, Yore MM, Dwyer PM, Syed I, Aryal P, Kahn BB. RBP4 activates antigen-presenting cells, leading to adipose tissue inflammation and systemic insulin resistance. Cell Metab. 2014;19:512-26.

7. Fasshauer M, Blüher M. Adipokines in health and disease. Trends Pharmacol Sci. 2015;36:461-70.

8. Kim SH, Chung JH, Song SW, Jung WS, Lee YA, Kim HN. Relationship between deep subcutaneous abdominal adipose tissue and metabolic syndrome: a case control study. Diabetol Metab Syndr. 2016;8:10.

9. Esfahani M, Movahedian A, Baranchi M, Goodarzi MT. Adiponectin: an adipokine with protective features against metabolic syndrome. Iran J Basic Med Sci. 2015;18:430-42.

10. Turer AT, Scherer PE. Adiponectin: mechanistic insights and clinical implications. Diabetologia. 2012;55:2319-26.

11. Daigo K, Mantovani A, Bottazzi B. The yin-yang of long pentraxin PTX3 in inflammation and immunity. Immunol Lett. 2014;161:38-43.

12. Inoue K, Kodama T, Daida H. Pentraxin 3: a novel biomarker for inflammatory cardiovascular disease. Int J Vasc Med. 2012;2012:657025.

13. Yamasaki K, Kurimura M, Kasai T, Sagara M, Kodama T, Inoue K. Determination of physiological plasma pentraxin 3 (PTX3) levels in healthy populations. Clin Chem Lab Med. 2009; 47:471-7.

14. Hasan A, Al-Ghimlas F, Warsame S, Al-Hubail A, Ahmad R, Bennakhi A, Al-Arouj M. IL-33 is negatively associated with the $\mathrm{BMI}$ and confers a protective lipid/metabolic profile in nondiabetic but not diabetic subjects. BMC Immunol. 2014;15:19.

15. Miller AM. Role of IL-33 in inflammation and disease. J Inflamm (Lond). 2011;8:22.

16. Grundy SM, Cleeman JI, Daniels SR, Donato KA, Eckel RH, Franklin BA, et al.; American Heart Association; National Heart, Lung, and Blood Institute. Diagnosis and management of the metabolic syndrome: an American Heart Association/National Heart, Lung, and Blood Institute Scientific Statement. Circulation. 2005;112:2735-52.

17. Rizk FH, Elshweikh SA, Abd El-Naby AY. Irisin levels in relation to metabolic and liver,ctions in Egyptian patients with metabolic syndrome. Can J Physiol Pharmacol. 2015:1-4.

18. García-Fontana B, Reyes-García R, Morales-Santana S, ÁvilaRubio V, Muñoz-Garach A, Rozas-Moreno P, et al. Relationship between myostatin and irisin in type 2 diabetes mellitus: a compensatory mechanism to an unfavourable metabolic state? Endocrine. 2016;52:54-62. 
19. Park KH, Zaichenko L, Brinkoetter M, Thakkar B, Sahin-Efe $A$, Joung $K E$, et al. Circulating irisin in relation to insulin resistance and the metabolic syndrome. J Clin Endocrinol Metab. 2013;98:4899-907.

20. Assyov Y, Gateva A, Tsakova A, Kamenov Z. Irisin in the Glucose Continuum. Exp Clin Endocrinol Diabetes. 2016;124:22-7.

21. Wang W, Li N. Correlation of retinol binding protein 4 with metabolic indexes of glucose and lipid, bile cholesterol saturation index. Zhong Nan Da Xue Xue Bao Yi Xue Ban. 2015;40:657-65.

22. Duran ID, Gülçelik NE, Ünal M, Topçuoğlu C, Sezer S, Tuna MM, et al. Irisin levels in the progression of diabetes in sedentary women. Clin Biochem. 2015;48:1268-72.

23. Trayhurn P,Wood IS. Adipokines: inflammation and the pleiotropic role of white adipose tissue. Br J Nutr. 2004;92:347-55.

24. Kardas F, Akın L, Kurtoglu S, Kendirci M, Kardas Z. Plasma Pentraxin 3 as a biomarker of metabolic syndrome. Indian $\mathrm{J}$ Pediatr. 2015;82:35-8.

25. Zanetti M, Bosutti A, Ferreira C, Vinci P, Biolo G, Fonda M, et al. Circulating pentraxin 3 levels are higher in metabolic syndrome with subclinical atherosclerosis: evidence for association with atherogenic lipid profile. Clin Exp Med. 2009;9:243-8.

26. Wang L, Song J, Wang C, Lin P, Liang K, Sun Y, et al. Circulating levels of betatrophin and irisin are not associated with pancreatic $\beta$-cell function in previously diagnosed type 2 diabetes mellitus patients. J Diabetes Res. 2016;2016:2616539.
27. Park SE, Lee NS, Park JW, Rhee EJ, Lee WY, Oh KW, et al. Association of urinary RBP4 with insulin resistance, inflammation, and microalbuminuria. Eur J Endocrinol. 2014;171:443-9.

28. Ingelsson E, Sundström J, Melhus $H$, Michaëlsson K, Berne C, Vasan RS, et al. Circulating retinol-binding protein 4, cardiovascular risk factors and prevalent cardiovascular disease in elderly. Atherosclerosis. 2009;206:239-44.

29. Feng S, Zhu Y, Yan C, Wang Y, Zhang Z. Retinol binding protein 4 correlates with and is an early predictor of carotid atherosclerosis in type 2 diabetes mellitus patients. J Biomed Res. 2015 Jul 3;29. doi: 10.7555/JBR.29.20140087. [Epub ahead of print].

30. Jialal I, Adams-Huet B, Duong F, Smith G. Relationship between retinol-binding protein-4/adiponectin and leptin/adiponectin ratios with insulin resistance and inflammation. Metab Syndr Relat Disord. 2014;12:227-30.

31. Zhang M, Chen P, Chen S, Sun $Q$, Zeng QC, Chen JY, et al. The association of new inflammatory markers with type 2 diabetes mellitus and macrovascular complications: a preliminary study. Eur Rev Med Pharmacol Sci. 2014;18:1567-72.

32. Bidulescu A, Liu J, Hickson DA, Hairston KG, Fox ER, Arnett DK, et al. Gender differences in the association of visceral and subcutaneous adiposity with adiponectin in African Americans: the Jackson Heart Study. BMC Cardiovasc Disord. 2013;13:9.

33. Chiara TD, Argano C, Scaglione A, Corrao S, Pinto A, Scaglione R. Circulating adiponectin: a cardiometabolic marker associated with global cardiovascular risk. Acta Cardiol. 2015;70:33-40. 